\title{
Performance and Processing of SAR Satellite Clusters
}

\author{
Jim Stiles, Nathan Goodman, and SiChung Lin \\ The University of Kansas, Radar Systems and Remote Sensing Laboratory \\ 2291 Irving Hill Road, Lawrence, KS 66044 \\ Phone: (785) 864-7744, Fax: (785) 864-7789, E-mail: jstiles@ rsl.ukans.edu
}

\begin{abstract}
Spaceborne radar systems have been proposed that use the concept of "formation flying" satellites; a system wherein a cluster of small, individual satellites orbit the Earth while remaining in the same relative positions with respect to each other. Each individual satellite would be a standard SAR sensor, but the data collected from each could be combined to produce a single "virtual" satellite from the overall array of sensors. The question from a radar standpoint is, how should this data be processed, and what are the benefits in radar performance resulting from this design?

A spaceborne array of radar satellites has the advantage that angle-of-arrival information, in addition to rangeDoppler data, is collected. Essentially, the array of satellites can be viewed as a large, albeit sparse, array of antennas. This paper demonstrates that this extra information can be used to produce SAR imagery over both an arbitrarily wide swathwidth and with fine resolution. This is accomplished by applying a minimum mean squared error processing algorithm to combine the data from each sensor.

The results of several simulations are shown; they demonstrate the efficacy of the concept and the processing. It is also shown that, as the number of satellites increases, the performance of the MMSE processing increases and the hardware requirements associated with each radar are eased.
\end{abstract}

\section{INTRODUCTION}

A fundamental limitation on the utility of SAR sensors is the limitation on image area, or swadthwidth [1,2]. Recently, sensors have been proposed that have multiple receive apertures, each with a coherent receiver [3]. As a result, many more independent samples are collected over a given time. These independent samples contain spatial information about target angle of arrival, in addition to standard delayDoppler responses. This additional information can be used to extend the image area/swadthwidth, as well as improve the quality of the overall SAR image.

\section{BACKGROUND}

A radar illuminating an area $A$ of the Earth's surface will receive a complex response $\mathrm{r}(\mathrm{t})$ described as:

$$
\begin{aligned}
r(t) & =\iint_{0} \gamma_{0}(\bar{x}) h\left(\bar{x}, t, t^{\prime}\right) s\left(t^{\prime}\right) d t^{\prime} d A+n(t) \\
& =\int_{A} \gamma_{0}(\bar{x}) \rho(\bar{x}, t) d A+n(t)
\end{aligned}
$$

where $\bar{x}$ is a position vector describing surface location, $\gamma_{0}(\bar{x})$ is the complex scattering coefficient of the surface, $h\left(\bar{x}, t, t^{\prime}\right)$ is a complex, time-varying impulse response describing the propagation from the moving radar to the surface and back, and $s(t)$ is a complex transmit signal. Since this transmit signal is constrained both in bandwidth and in time, it can be approximated with discrete samples as:

$s(t) \cong \sum_{n}^{N} s\left(t_{n}\right) g\left(t-t_{n}\right)$

The benefit of this representation is that the radar response can be represented using linear algebra:

$$
\begin{array}{ll}
\mathbf{r}=\sum_{i} \gamma_{i} \mathbf{H}^{i} \mathbf{S}+\mathbf{n}=\sum_{i} \gamma_{i} \boldsymbol{\rho}_{i}+\mathbf{n} \\
\\
\text { where } \quad r_{m}=r\left(t_{m}\right) \\
\gamma_{i}=\gamma_{0}\left(\bar{x}_{i}\right) \Delta A \\
H_{m n}^{i}=\int h\left(\bar{x}_{i}, t_{m}, t^{\prime}\right) g\left(t^{\prime}-t_{n}\right) d t^{\prime} \\
s_{n}=s\left(t_{n}\right) \\
n_{m}=n\left(t_{m}\right)
\end{array}
$$

SAR processing is a linear operation; therefore we seek a vector operator $\mathbf{w}_{i}$ that works on $\mathbf{r}$ to produce an estimate of $\gamma_{i}$, the scattering coefficient for resolution cell $i$ (i.e., $\left.\hat{\gamma}_{i}=\mathbf{w}_{i}^{\prime} \mathbf{r}\right)$. More generally, we seek a matrix operator $\mathbf{W}$ such that $\hat{\boldsymbol{\gamma}}=\mathbf{W r}$, where $\hat{\boldsymbol{\gamma}}=\left[\begin{array}{lll}\hat{\gamma}_{1} & \cdots & \hat{\gamma}_{I}\end{array}\right]^{T}$ and $\mathbf{W}=\left[\begin{array}{lll}\mathbf{w}_{1} & \cdots & \mathbf{w}_{l}\end{array}\right]^{T}$. The dimensions of the two vectors determine the difficulty of this problem. If the dimension of $\boldsymbol{\gamma}$ exceeds the dimension of $\mathbf{r}$, then the number of independent samples representing the signal measurement is less than the number of resolution cells (i.e., pixels) to be estimated. The result is dependent estimates of $\boldsymbol{\gamma}$ and thus a distorted SAR map. The dimension of measurement vector $\mathbf{r}$ is set by the time bandwidth product $B T$ of the received signal, whereas the dimension of $\boldsymbol{\gamma}$ (the number of illuminated pixels) is set by the resolution of the radar and the illumination area $A$. However, the range and Doppler resolution of SAR is also 
determined by the bandwidth $B$ and timewidth $T$ of the signal. Therefore, the dimension of $\mathbf{r}$ will exceed that of $\boldsymbol{\gamma}$ only if the illuminaton area $A$ is restricted, resulting in a minimum aperture size for SAR sensors.

\section{MULTI-APERTURE SAR}

Increasing either the timewidth $T$ or bandwidth $B$ does increase the dimension of measurement vector $\mathbf{r}$, and thus allows for additional unambiguous pixels to be imaged. However, since the resolution of the sensor likewise increases, more pixels are resolved within the illumination area $A$, but the size of $A$ is still restricted. In order to increase illumination area $A$ without affecting resolution, additional independent samples must be collected without modifying $T$ or $B$.

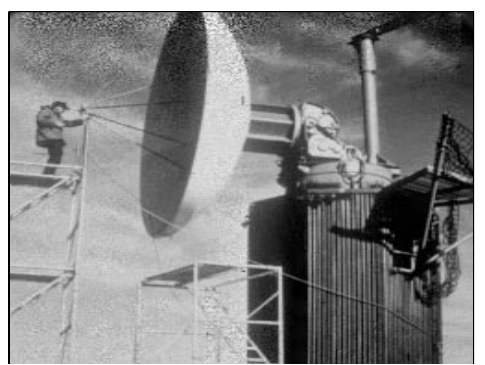

Figure 1: Correlation filter processing on multi-aperture SAR.

This can be accomplished by increasing the number of receive apertures associated with each SAR. For example, a SAR aperture could be divided into two smaller apertures, each with a coherent receiver. The receive apertures would illuminate twice as much area; however they would also collect twice as much data, each aperture collecting $B T$ independent samples. As a result, the maximum surface area (swathwidth) that can be imaged is twice that of a single-
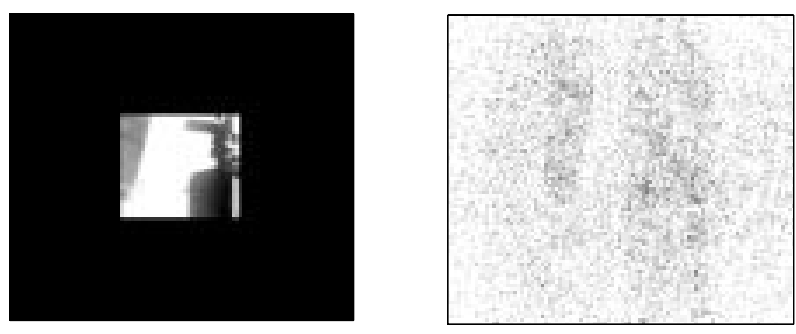

Figure 2: Single-aperture SAR results, demonstrating choice between restricted image area or large ambiguous imaged area.

aperture SAR. As the number of apertures is increased, the image area, or swathwidth, likewise increases. The only requirement is that the total receive aperture area exceeds the value required for single-aperture SAR.

The problem therefore, is how to process this additional received data; in other words, to find the linear SAR operator W. Generally, this linear operator is a correlation, or matched filter, wherein the wieght vector $\mathbf{w}_{i}$ depends on the response from resoultion cell $i$ only:

$\mathbf{w}_{i}=\frac{\boldsymbol{\rho}_{i}}{\left|\boldsymbol{\rho}_{i}\right|^{2}}$

Fig. 1 shows the result of this processing on a simulated scattering image. The number of pixels in the image exceeds the timebandwidth product of the received signal by nine times, therefore the receive aperture was subdivided into 11 sections. By contrast, Fig. 2 shows this same image using single-aperture SAR-either the aperture size must be restricted, resulting in an unambiguous but restricted image, or the aperture size is increased to illuminate the entire area, resulting in an ambiguous image.

The correlation filter results in the large unambiguous image of Fig. 1 because it operates on all the received data. For a single aperture SAR, this forms a traditional range Doppler map. For a multi-aperture SAR, however, additional data is collected as angle-of-arrival information across the receive array. As a result, ambiguities in range and Doppler are effectively resolved in angle of arrival. The correlation filter essentially performs digital beamforming on the spatial data, such that ambiguous targets in the range-Doppler map are suppressed.

The scattering estimate for a given pixel using a correlation filter is:

$\hat{\gamma}_{i}=\gamma_{i}+\sum_{i \neq j} \gamma_{j} \frac{\boldsymbol{\rho}_{i}^{\prime} \boldsymbol{\rho}_{j}}{\left|\boldsymbol{\rho}_{i}\right|^{2}}+\frac{\boldsymbol{\rho}_{i}^{\prime} \mathbf{n}}{\left|\boldsymbol{\rho}_{i}\right|^{2}}$

This equation demonstrates the problem with using matched filtering in SAR. The first term is the desired estimate, and therefore the final two terms are error values. The second is the contribution due to clutter, while the third is the contribution due to noise. The matched filter, of course, minimizes the effect of noise, but does nothing to affect clutter. This is particularly a problem when considering multi-aperture distributed SAR. The receive apertures of a multi-aperture SAR are not required to be contiguous. Recently, systems have been considered that would sparsely distribute apertures across an extent of several hundred meters, such that each aperture constitutes an individual satellite. This collection of satellites would thus create a single, multi-aperture SAR. However, applying the matched filter to a sparse array causes results as shown in Fig. 3. The beamforming sidelobes of the sparse array are generally large, and thus do a poor job of suppressing pixels ambiguous in range and Doppler. In this case, target clutter is the major problem. 


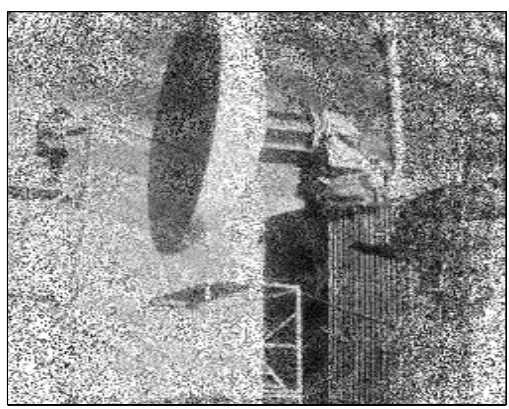

Figure 3: Correlation filter processing on distributed 13-aperture SAR.

An operator $\mathbf{W}$ does exist that minimizes the effect of clutter. Ignoring the noise term in (3), it is evident that an estimate of $\boldsymbol{\gamma}$ can formed as:

$$
\hat{\gamma}=\mathbf{P}^{\sim 1} \mathbf{r}
$$

where $\mathbf{P}=\left[\boldsymbol{\rho}_{1}, \boldsymbol{\rho}_{2}, \ldots \boldsymbol{\rho}_{1}\right]$. The inverse in this case is a pseudo inverse, since the dimension of $\mathbf{r}$ exceeds that of $\boldsymbol{\gamma}$. This is essentially a deconvolution operation, and generally results in inferior performance for the single-aperture SARs. However, for multi-aperture SAR, the dimension of the measurement vector $\mathbf{r}$ can significantly exceed that of $\boldsymbol{\gamma}$, so that the matrix $\mathbf{P}^{-1}$ is generally well conditioned. The linear operator $\mathbf{W}=\mathbf{P}^{-1}$ gives significantly improved performance for cases where SNR is high, as shown in Fig. 4. This processor finds a weight vector that is orthogonal to the responses of all other pixel targets, and thus the second term ( the clutter term) of equation (5) is ideally zero.

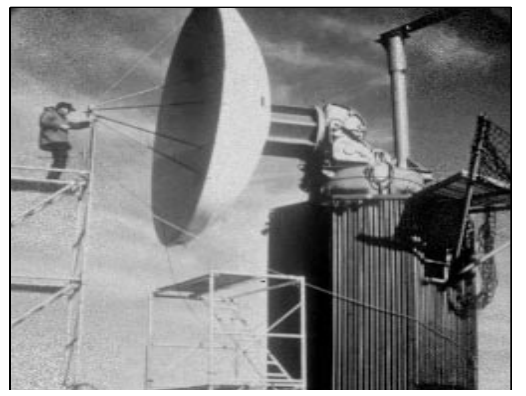

Figure 4: Orthogonal filter processing on distributed-aperture SAR.

However, since this orthogonal operator does nothing to minimize noise, the processing performance diminishes as SNR drops. An ideal processor therefore would maximize the Signal-to-Interference Ratio (SIR), where interference is defined as the sum of both clutter and noise energy. This processor is the Minimum Mean Squared Error (MMSE) solution, given as:

$$
\begin{aligned}
\mathbf{W}_{\text {mmse }}=\sigma_{\gamma}^{2} \mathbf{P}^{\prime} & {\left[\mathbf{P P}^{\prime} \sigma_{\gamma}^{2}+\sigma_{n}^{2} \mathbf{I}\right]^{-1} } \\
\sigma_{\gamma}^{2} & =\text { Expected value of }|\gamma|^{2} \\
\text { where } \quad \sigma_{n}^{2} & =\text { Noise variance } \\
\mathbf{I} & =\text { Identity matrix }
\end{aligned}
$$

Fig. 5 shows the estimate error for a distributed, multiaperture design as a function of SNR, for each of the three processors discussed in this paper. The result demonstrates that the MMSE is the superior processor regardless of SNR.

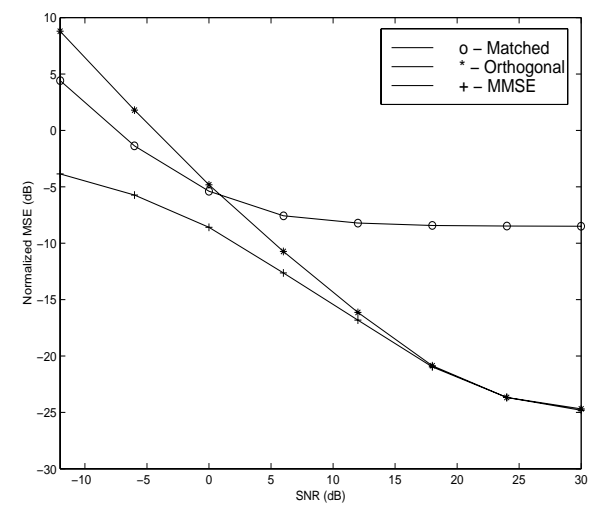

Figure 5: Image error vs. SNR for all three processing operators.

\section{CONCLUSIONS}

Multi-aperture SAR collects allows for increased image area or swathwidth. Additionally, image quality is increased if MMSE processing is implemented. This processing is required for distributed receiver arrays, as correlation processors generate unsatisfactory image results. Additional receive apertures increase the dimension of receive vector $\mathbf{r}$ over that of scattering vector $\boldsymbol{\gamma}$, with a corresponding improvement in SAR image quality.

\section{REFERENCES}

[1] F. K. Li and W. T. K. Johnson, "Ambiguities in Spaceborne Synthetic Aperture Radar Systems," IEEE Trans. Aerosp. Electron. Syst., vol AES-19, no. 3, pp. 389-396, May 1983.

[2] K. Tomiyasu, "Image Processing of Synthetic Aperture Radar Range Ambiguous Signals," IEEE Trans. Geosci. Remote Sensing, vol. 32, no. 5, pp-1114-1117, Sep. 1994.

[3] M. Younis and W. Wiesbeck, "SAR with Digital Beamforming on Receive Only," Proc. IGARSS '99, vol. 3, pp. 1773-1775. 